May 2006

\title{
Probability Modeling of Autonomous Unmanned Combat Aerial Vehicles (UCAVs)
}

by

\author{
Moshe Kress $^{1^{*}}$ \\ Arne Baggesen ${ }^{2}$ \\ Eylam Gofer ${ }^{3}$
}

${ }^{1}$ Operations Research Dept., Naval Postgraduate School, Monterey CA.

${ }^{2}$ MOVES Institute, Naval Postgraduate School, Monterey CA, and German Naval Office, Rostock, Germany

${ }^{3}$ Center for Military Analyses, Haifa, Israel

${ }^{*}$ To whom correspondence should be addressed. E-mail: $\underline{\text { mress@nps.edu }}$

APPLICATION AREA: Unmanned Systems, Strike Warfare.

OR METHODOLOGY: Markov Processes 


\begin{abstract}
Unmanned Combat Aerial Vehicles (UCAVs) are advanced weapon systems that can loiter autonomously in a pack over a target area, detect and acquire the targets, and then engage them. Modeling these capabilities in a specific hostile operational setting is necessary for addressing weapons' design and operational issues. In this paper we develop several analytic probability models, which range from a simple regenerative formula to a large-scale continuous-time Markov chain, with the objective to address the aforementioned issues. While these models capture key individual aspects of the weapon such as detection, recognition, memory and survivability, special attention is given to pack related aspects such as simultaneous targeting, multiple kills due to imperfect battle damage assessment, and the effect of attack coordination. From implementing the models we gain some insights on design and operational considerations regarding the employment of a pack of UCAVs in a strike scenario.
\end{abstract}




\section{INTRODUCTION}

Advances in sensors and command, control, communications, computers, intelligence, surveillance and reconnaissance (C4ISR) technologies, coupled with operational needs, like the war against terror, have led in recent years to the development of a new class of weapon systems called Unmanned Combat Aerial Vehicles, or in short UCAVs. A UCAV is a self-propelled aerial vehicle that typically loiters over the target area, seeking targets for engagement. UCAVs combine a unique set of capabilities in one platform; they have an eye that senses the area and gathers target information, a brain that processes this information, wings that move the UCAV around and keep it aloft and a fist, in a form of a warhead. There are two major types of UCAVs: disposable and retrievable. Disposable UCAVs are essentially precision guided munitions (PGM), like guided missiles, where the warhead is an integral part of the platform. Thus, a UCAV of this type can engage at most one target. Examples of disposable UCAVs are the Israeli Harpy (Jane’s, 2000a), the German Taifun (Jane's, 2000b) and the US (Lockheed Martin) LOCAAS (Jane’s, 2002). Retrievable UCAVs are larger vehicles that carry one or more munitions, which are launched from the vehicle towards the targets in a controlled trajectory. Once the weapons are expended, the UCAV returns to its base for refit and reload. An example of a retrievable UCAV is the US Air force Predator that can carry a Hellfire laser-guided missile (Airforce Technology 2005).

In this paper we focus on autonomous UCAVs, which are designed to operate as a pack of vehicles that autonomously search, detect, acquire and attack targets. Similar operational concepts are imbedded in the Autonomous Wide Area Search Munition (AWASM), which is developed by Lockheed Martin for the US Air Force (Lockheed Martin, 2004). While much attention is given to the engineering and technological aspects of UCAV developments, there are very few studies on operational concepts for these weapon systems and their expected effectiveness and efficiency. The wide range of design and operational factors and capabilities of such autonomously acting and interacting weapons will most likely lead to a wide range of engagement performance in various scenarios. The problems are to select proper measures of effectiveness (MOEs) for the engagement performance, map the functional relations 
between the parameters and the MOEs, and obtain insights regarding the design of the UCAVs and their tactical employment.

While target detection and recognition capabilities, and weapon's accuracy and lethality determine the effectiveness of a single vehicle, two phenomena may affect the performance of the UCAVs as a pack: multiple acquisitions and multiple kills. Multiple acquisitions occur when two or more UCAVs acquire, and are about to engage, the same target. This situation, which may lead to redundancy and waste of attack resources, is due to lack of targeting coordination among the UCAVs. Absent multiple acquisitions, multiple kills occur when a UCAV engages a target that has already been killed by another UCAV. This situation is due to imperfect battle damage assessment (BDA).

The issue of coordination and cooperative control for target acquisition is addressed in several studies. Jacques (2002) presents a simple probability model for examining some operational aspects of employing a pack of AWASM. Other studies (e.g., Chandler et al, (2002), Gillen and Jacques (2002) and Richards et al (2002)) utilize simulations for evaluating possible information sharing schemes, and develop optimization (mixed-integer programming) models that produce task assignment rules for target observation and classification and trajectory designs. Jeffcoat (2004) applies Markov-chain analysis to study the effect of cueing in the case of two cooperative searchers. The effect of BDA is analyzed in the context of Shoot-Look-Shoot models. Aviv and Kress (1997) utilize Markov and dynamic programming models to evaluate several shooting tactics when damage information is only partial. Manor and Kress (1997) prove the optimality of a certain shooting tactics under conditions of incomplete information. An optimal assignment of weapons and BDA sensors is presented in Yost and Washburn (2000), and a general review of probability models for evaluating Shoot-Look-Shoot models in the presence of partial damage information is given in Glazebrook and Washburn (2004).

In this paper we develop analytic probability models for analyzing some design and operational aspects relating to autonomous UCAVs. The models range from a simple regenerative formula to a large scale continuous-time Markov chain. In addition to considering individual UCAV properties - detection, recognition, memory, kill- 
effectiveness and vulnerability - the models explicitly incorporate also the effect of multiple acquisitions and multiple kills. Unlike simulations, a single run of each of these models produces exact probability distributions and values for the MOEs, and by applying these models to a set of design and operational parameters some insights - not all intuitive - are gained. The framework of the paper The rest of the paper is organized as follows. In Section 2 we describe the basic operational setting of the situation we model, and in Section 3 we introduce notation and discuss the basic assumptions. In Section 4 we address the issue of UCAV memory and answer the question “is it an important feature?” Some transient properties of the engagement process in the case of no situational awareness are presented in Section 5. In Section 6 we study the complete problem where both multiple acquisition and situational awareness are considered. We formulate the continuous-time Markov model and present the results of the analysis, along with some design and operational insights. Summary and concluding remarks are presented in Section 7.

\section{THE BASIC SITUATION}

A pack of single-weapon autonomous unmanned combat aerial vehicles (UCAV) is launched on a mission to attack a set of homogeneous targets located on the ground or at sea in a specific target area. Each UCAV loiters independently over the target area searching for valuable targets. The definition of a valuable target depends on the scenario and mission e.g., armored vehicles in tactical ground combat scenarios, airdefense missile launchers and radar sites in suppression of air defense (SEAD) missions, and command posts in operational-level missions. All other targets are nonvaluable. A killed valuable target becomes non-valuable.

During its mission, a UCAV can be in one of three possible situations: search, attack or removed. A UCAV is said to be searching if it is still loitering and it has not acquired a target for engagement yet. Once a UCAV detects a target it locks on the target and attempts to identify if it is a valuable or non-valuable target. If the UCAV classifies the target (correctly or incorrectly) as non-valuable, the target is rejected (not acquired), the UCAV disengages and moves on with its search. If the UCAV classifies a target as valuable, it acquires the target and attacks it. The randomly distributed inter-detection time of a UCAV in a search stage is defined as the time between two consecutive detections of targets. This time comprises the loitering time 
from the last rejection to a new detection, and the identification time between the moment of lock-on and the moment the UCAV identifies the target and decides to attack (in case of acquisition) or disengage (in case of rejection). The total search time of a UCAV is the sum of its inter-detection times. We assume that the inter-detection times are not dependent on the classification result. The randomly distributed attack time is measured from the moment the target is classified as valuable to the moment the weapon hits the ground (or the surface). Figure 1 describes the aforementioned mission time parameters.

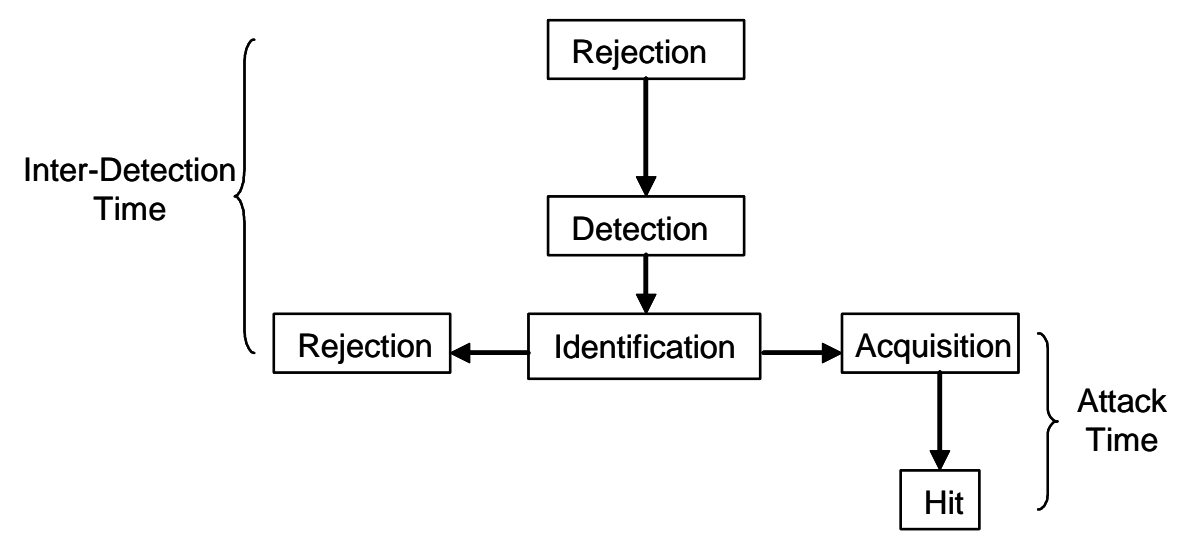

Figure 1: UCAV Mission Timeline

Once a UCAV enters an attack stage, it is committed to attack the acquired target and therefore cannot go back to the search stage, even if during the time of the attack another UCAV hits the target and kills it. Thus, if several UCAVs acquire the same target, at most one of them can be effective. We consider a UCAV that is either disposable or carries a single missile, therefore after an attack the UCAV is removed from further consideration in the current mission. A UCAV may fail during the search or attack stages if it is intercepted by enemy's air defense or it crashes due to technical failure or accident. We assume imperfect sensitivity and specificity; therefore identification may be subject to error. A valuable target may be identified, due to imperfect sensitivity, as non-valuable and therefore passed over by the UCAV, and a non-valuable target may be identified, due to imperfect specificity, as valuable and therefore attacked by the UCAV. We assume that the nominal loitering time (e.g., due to fuel consumption) is long compared to the minimum between the time it takes a 
UCAV to acquire and attack a target and the time until it (possibly) fails. In other words, a UCAV never runs out of fuel before its mission is over.

Given this combat situation, we wish to measure the effectiveness of the UCAVs, perform sensitivity analysis, and determine tradeoffs among design and operational parameters.

\section{NOTATION AND BASIC ASSUMPTIONS}

The probabilities of correctly identifying a valuable target and correctly identifying a non-valuable target are $q_{1}$ and $q_{2}$, respectively. That is, $q_{1}$ represents the sensitivity of the UCAV's sensor and data processing unit, and $q_{2}$ their specificity. The identification attempts are independent. The sensitivity and specificity of a UCAV determine its BDA capabilities. BDA (battle damage assessment) refers to the ability of a shooter to distinguish between a live valuable target and a killed one (which becomes non-valuable). For simplicity we assume that the specificity of the UCAV with respect to initially non-valuable targets is the same as for killed valuable targets. The models can be easily generalized to account for target dependent specificity. An acquired target is successfully hit and killed with probability $p$. To simplify the model, and without loss of generality, we assume that the probability of a kill given a hit is 1 . We assume that the inter-detection and the attack times are exponentially distributed random variables with parameters $\lambda$ and $\mu$, respectively. While the former is a reasonable assumption based on the independent and memory-less nature of the search process (see Section 4 below), the latter is an approximation, which is similar to the exponential inter-firing assumption in stochastic duel or stochastic Lanchester models (e.g., Kress (1991) and Kress and Talmor (1999)). The failure rate of UCAVs is assumed to be constant and therefore the time until a UCAV fails is exponentially distributed random variable with parameter $\theta$. The launched pack comprises $N$ UCAVs. The total number of targets - valuable and non-valuable - in the target zone at the beginning of the operation is $T$, out of which $L$ targets are valuable and $T-L$ targets are non-valuable. 


\section{DOES MEMORY MATTER?}

Consider a single UCAV, which detects a target and decides, correctly or incorrectly, to reject it. This event may or may not register in the UCAV's memory. If the UCAV remembers the rejected targets, then it would not consider any of them for future acquisition and therefore, after a finite number of detections, the pool of potential targets for engagement may be depleted. Absent memory, and since the detections are independent, it is possible that the UCAV will acquire a previously rejected target. The question is, can memory enhance (or reduce) the probability that the search process terminates with a killed valuable target?

First we assume no memory (NM). That is, the UCAV may detect and examine the same target more than once. The probability $P_{N M}(T, L)$ that a UCAV acquires and kills a valuable target, given there are a total of $T$ targets and $L$ valuable targets in the target area, satisfies the following regenerative equation:

$$
P_{N M}(T, L)=\underbrace{\frac{\lambda}{\lambda+\theta}}_{\begin{array}{c}
\text { Probability of successful } \\
\text { detection }
\end{array}} \cdot[\underbrace{\frac{\mu}{\mu+\theta} \cdot \frac{L}{T} \cdot q_{1} \cdot p}_{\begin{array}{c}
\text { Probability of } \\
\text { successful attack }
\end{array}}+\underbrace{\left(\frac{L}{T} \cdot\left(1-q_{1}\right)+\frac{T-L}{T} \cdot q_{2}\right)}_{\text {Probability the target is rejected }} \cdot P_{N M}(T, L)] .
$$

The solution of (1) is:

$$
P_{N M}(T, L)=P(\alpha)=\frac{\mu}{\mu+\theta} \cdot \frac{\alpha \cdot \lambda \cdot q_{1} \cdot p}{\lambda+\theta \cdot(1+\alpha)+\alpha \cdot \lambda \cdot q_{1}-\lambda \cdot q_{2}}
$$

where $\alpha=L /(T-L)$. That is, the acquisition probability depends on the ratio between the numbers of valuable and non-valuable targets and not on their absolute numbers. Also it depends on the endurance ratios $\lambda / \theta$ and $\mu / \theta$, and not on the absolute values of the detection, attack and failure intensities.

Suppose now an ideal situation where the UCAV has perfect memory and situational awareness and therefore it would always detect and examine a new target. In that situation it is possible that the search process will terminate with no acquisition. In that case, we assume that the UCAV instantaneously selects any of the $T$ targets at random and attacks it. This termination condition is appropriate in particular in time- 
critical missions. Since previously detected targets are automatically discarded from the search process, the rate at which new targets are detected decreases as the number of detected targets increases. Specifically, if the nominal detection rate at the beginning of the operation is $\lambda$ then after $k$ detected (and rejected) targets, the rate at which new targets are detected is $\lambda(1-k / T)$. The probability of killing a valuable target $P_{M}(T, L)$ is

$$
\begin{array}{r}
P_{M}(T, L)=\underbrace{q_{1} \cdot p \cdot \frac{\mu}{\mu+\theta} \cdot \sum_{i=0}^{L-1 T-L} \sum_{j=0}^{\left(\begin{array}{c}
L \\
i
\end{array}\right)\left(\begin{array}{c}
T-L \\
j
\end{array}\right)} \cdot \frac{L-i}{T-(i+j)} \cdot \prod_{k=0}^{i+j}\left(\frac{\lambda(1-k / T)}{\lambda(1-k / T)+\theta}\right) \cdot\left(1-q_{1}\right)^{i} \cdot q_{2}^{j}}_{\text {Probability that a target was successfully attacked in one of the } T \text { dtections }} \\
+\underbrace{\frac{\mu}{\mu+\theta} \cdot \frac{L}{T} \cdot p \cdot \prod_{k=0}^{T-1}\left(\frac{\lambda(1-k / T)}{\lambda(1-k / T)+\theta}\right) \cdot\left(1-q_{1}\right)^{L} \cdot q_{2}^{T-L}}_{\begin{array}{c}
\text { Probability that all } \\
\text { the target fortartans resulted in rejection and therefore randomly }
\end{array}}
\end{array}
$$

Suppose that the target area contains a total of 16 targets. We consider three target postures in which the proportion of valuable targets $\frac{L}{T}$ are $1 / 4$ (e.g., a section of armored vehicles), 1/2 (e.g., two sections) and 3/4 (e.g., a company). For each one of the target postures we consider two endurance ratios $\frac{\lambda}{\theta} ; 20$ (high survivability rate), and 3 (low survivability rate). We assume also two attack situations: slow execution where $\mu=\lambda$ and fast execution, where $\mu=10 \lambda$. In all the scenarios we assume that the hit probability given acquisition $p=1$, which means that $P$ is in fact the acquisition probability. Note that $p$ is a multiplicative factor that does not affect the relative effectiveness of the no memory and full memory cases. For each one of the twelve scenarios we evaluate the kill (acquisition) probability $P$ for various values of sensitivity probability $q_{1}$ and specificity probability $q_{2}$. Tables $\mathrm{A} 1-\mathrm{A} 3$ in the Appendix detail the results of the analysis for three target postures: $\frac{L}{T}=\frac{1}{4}, \frac{1}{2}, \frac{3}{4}$, respectively. Figures $2-4$ present the comparison between the no-memory (black 
lines) and full-memory (grey lines) cases for the three target postures $\left(\frac{L}{T}=\frac{1}{4}, \frac{1}{2}, \frac{3}{4}\right)$ with $\frac{\lambda}{\theta}=20$ and $\mu=10 \lambda$.

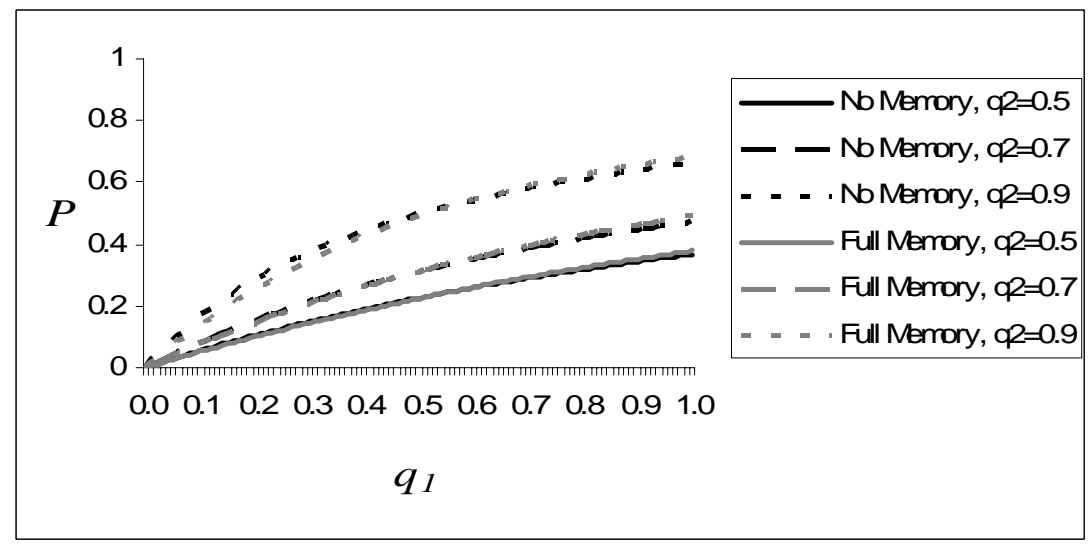

Figure 2: Probability of Acquiring a Valuable Target, $L / T=1 / 4$

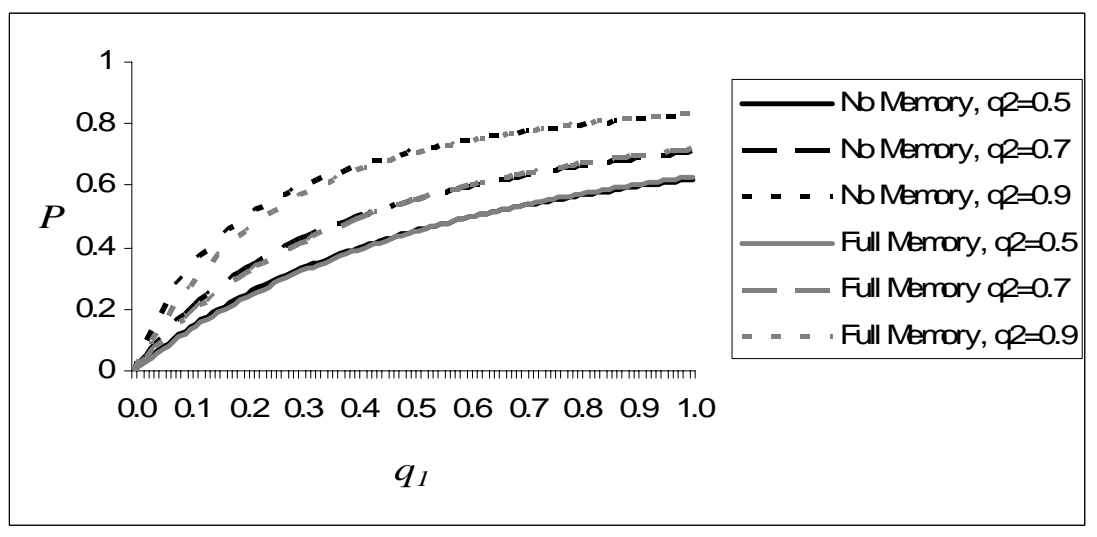

Figure 3: Probability of Acquiring a Valuable Target, $L / T=1 / 2$ 


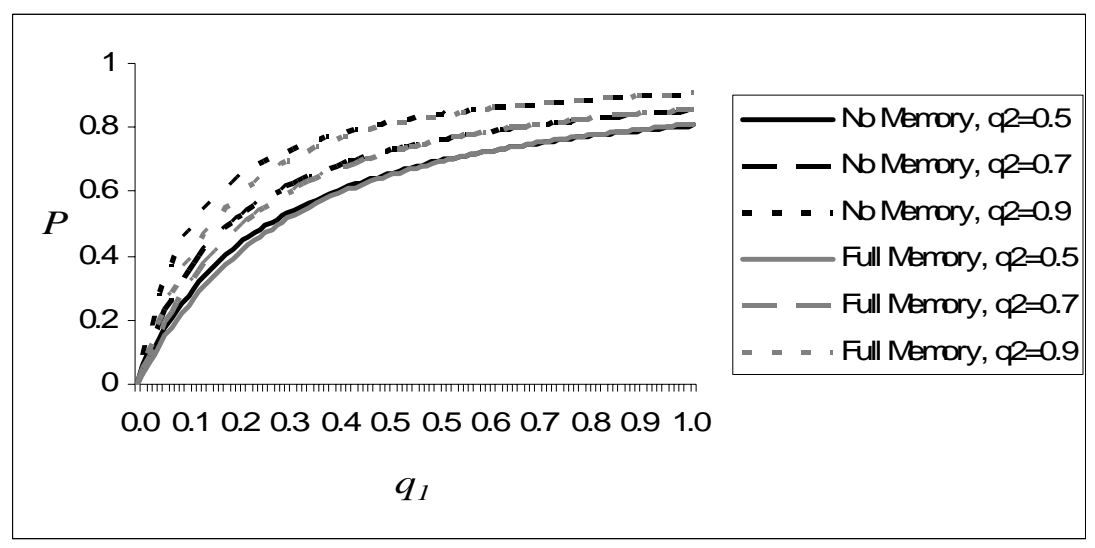

Figure 4: Probability of Acquiring a Valuable Target, $L / T=3 / 4$

Clearly, $P$ is monotonic increasing in both $q_{1}$ and $q_{2}$; better sensitivity and specificity results in higher acquisition probability. While for some (relatively small) values of $q_{1}$ and $q_{2}$ the no-memory system outperforms the full-memory system, and for other (relatively large) values the opposite is true, the differences between the two cases are negligible. This conclusion is robust with respect to the detection, attack and failure rates (see Appendix). For example, if $q_{1}=0.8$ and $q_{2}=0.7$ then the relative differences between $P_{M}$ and $P_{N M}$, over all twelve scenarios, range between $0 \%$ and less than 3\%. As shown in Tables A1-A3 in the appendix, this conclusion remains unchanged for longer inter-detection where $\lambda=\mu$.

Based on the analysis we can conclude that, under our assumptions, memory is rather redundant design feature in UCAVs. The processing capacity on board the UCAV would be better utilized for other data processing or storing tasks. Note however that this conclusion may not be true in other tactical settings such as time-critical missions or situations where the search time is limited due to operational or logistical constraints. From now on we assume that the UCAVs have no memory.

\section{MULTIPLE UCAVS, NO SITUATIONAL AWARENESS}

In this section we explore temporal effects of the UCAVs' target engagement process. We assume no situational awareness, which means that any detected target is attacked. In other words, $q_{1}=1-q_{2}=1$. 
The probability that at time $t$ of the engagement a certain UCAV is still searching is $e^{-(\lambda+\theta) t}$. Using conditioning, we obtain that the probability the UCAV failed by time $t$ is:

$$
\begin{aligned}
& Q_{F}(t)=\underbrace{\frac{\lambda \theta}{\mu+\theta} \int_{0}^{t} e^{-(\lambda+\theta) s}\left(1-e^{-(\mu+\theta)(t-s)}\right) d s}_{\text {Probability of failure during the attack stage }}+\underbrace{\theta \int_{0}^{t} e^{-(\lambda+\theta) s} d s}_{\begin{array}{c}
\text { Probability of failure } \\
\text { during the search stage }
\end{array}} \\
& =\left\{\begin{array}{c}
\frac{\lambda \theta}{\mu+\theta}\left[\frac{1-e^{-(\lambda+\theta) t}}{\lambda+\theta}-\frac{e^{-(\mu+\theta) t}}{\lambda-\mu}\left(1-e^{-(\lambda-\mu) t}\right)\right]+\frac{\theta}{\lambda+\theta}\left(1-e^{-(\lambda+\theta) t}\right) \text { if } \lambda \neq \mu \\
\frac{\lambda \theta}{\lambda+\theta}\left[\frac{1-e^{-(\lambda+\theta) t}}{\lambda+\theta}-t e^{-(\lambda+\theta) t}\right]+\frac{\theta}{\lambda+\theta}\left(1-e^{-(\lambda+\theta) t}\right) \quad \text { if } \lambda=\mu .
\end{array}\right.
\end{aligned}
$$

The probability that the UCAV has completed its mission by time $t$ without failure is

$$
\begin{aligned}
Q_{A}(t)= & \frac{\lambda \mu}{\mu+\theta} \int_{0}^{t} e^{-(\lambda+\theta) s}\left(1-e^{-(\mu+\theta)(t-s)}\right) d s= \\
& = \begin{cases}\frac{\lambda \mu}{\mu+\theta}\left(\frac{1-e^{-(\lambda+\theta) t}}{\lambda+\theta}-\frac{e^{-(\mu+\theta) t}\left(1-e^{-(\lambda-\mu) t}\right)}{\lambda-\mu}\right) & \text { if } \lambda \neq \mu \\
\frac{\lambda^{2}}{\lambda+\theta}\left(\frac{1-e^{-(\lambda+\theta) t}}{\lambda+\theta}-t e^{-(\lambda+\theta) t}\right) & \text { if } \lambda=\mu,\end{cases}
\end{aligned}
$$

and $Q_{A}(t) \underset{t \rightarrow \infty}{\rightarrow} \frac{\lambda \mu}{(\lambda+\theta)(\mu+\theta)}$.

Since the UCAVs are independent, the CDF of the duration of the operation is:

$$
F_{D}(t)=\left[Q_{F}(t)+Q_{A}(t)\right]^{N}
$$

and the expected number of killed targets at time $t$ is

$$
E_{t}=L\left(1-\left(1-\frac{Q_{A}(t) p}{T}\right)^{N}\right)
$$

Consider the base case where the average detection time is 5 minutes, the average attack time is 30 seconds and the mean time between failures (MTBF) is 100 minutes, 
that is, $\lambda=0.2, \mu=2$ and $\theta=0.01$. Figure 5 depicts the CDF of the operation completion time for various pack sizes $N$.

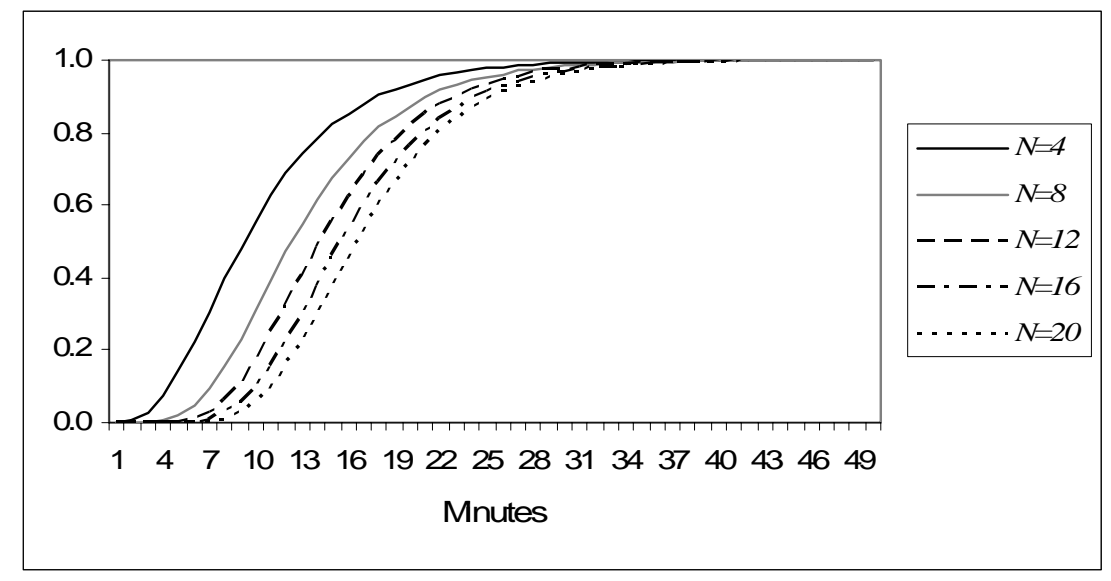

Figure 5: CDF of the Operation Completion Time for Varying $N$

The $90^{\text {th }}$ percentiles of these CDFs are 18, 23, 26, 27 and 28 minutes for packs of 4, 8, 12, 16 and 20 UCAVs, respectively. Figures 6 and 7 present the CDF of the mission completion time for varying detection intensities $(\lambda)$ and failure intensities $(\theta)$, respectively. In both cases we assume a pack of $N=8 \mathrm{UCAVs}$. The values of the other parameters are as in the base case.

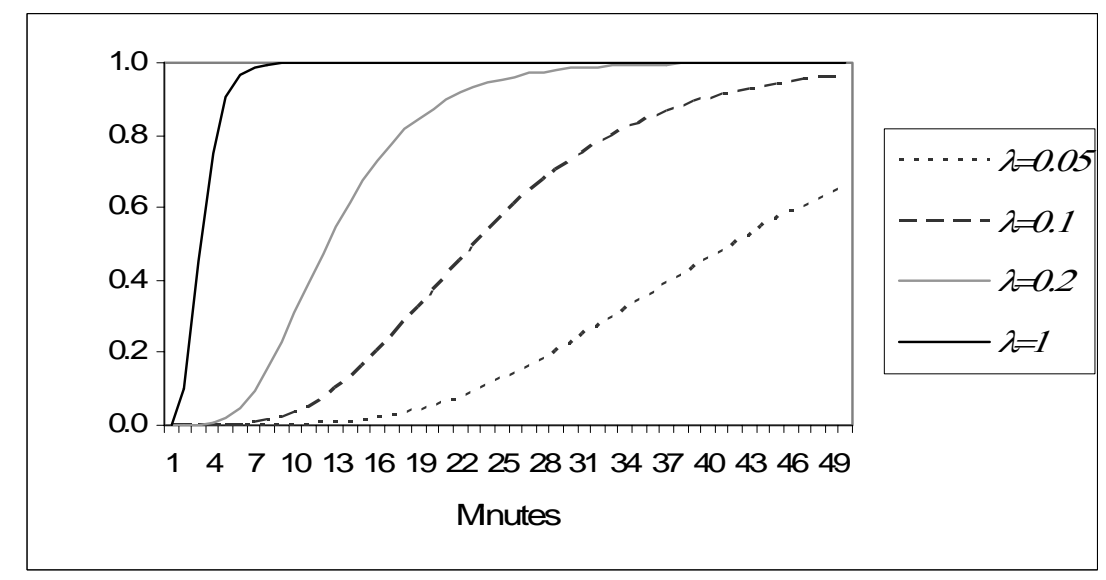

Figure 6: CDF of the Operation Completion Time for Varying $\lambda$

The $90^{\text {th }}$ percentiles of these distributions are $72,40,21$, and 5 minutes for mean detection times of 20,10, 5 and one minutes, respectively. 


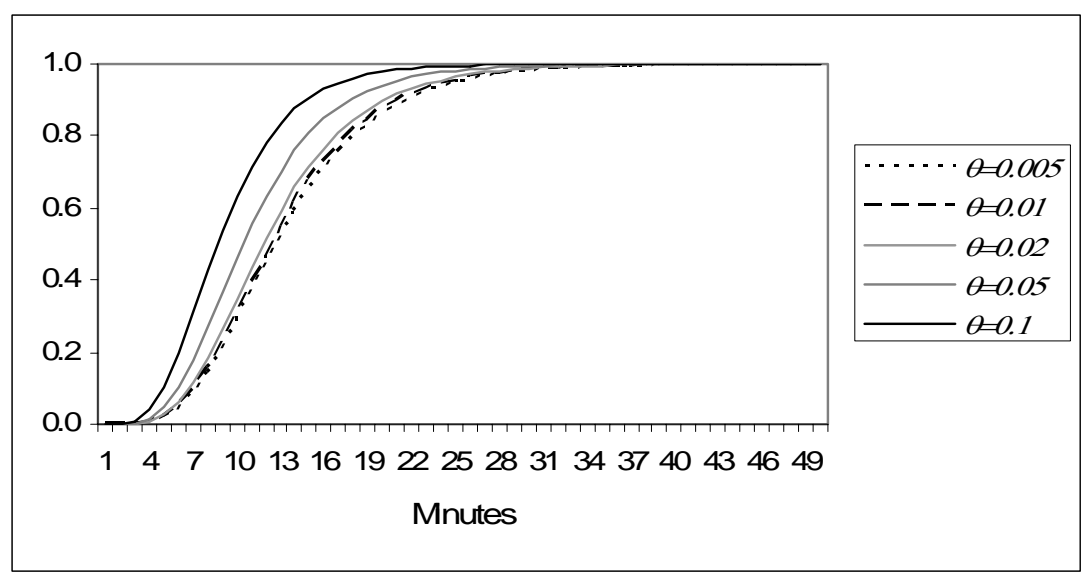

Figure 7: CDF of the Operation Completion Time for Varying $\theta$

The $90^{\text {th }}$ percentiles of the CDFs in Figure 5.3 are 22, 21, 20, 18 and 14 minutes for mean interception times of 200, 100, 50, 20 and 10 minutes, respectively. While the completion time of the mission is sensitive to the pack size and very sensitive to the detection intensity, it is rather insensitive to the failure rate within the relevant range. In other words, for the selected ranges of the time parameters, the most significant factor is the detection time.

Figure 8 shows the expected number of killed targets, out of an initial cluster of $L=T$ $=15$ targets (i.e., all targets are initially valuable), as a function of time. For $\lambda, \mu$ and $\theta$ we assume the base case and $N=8$ UCAVs.

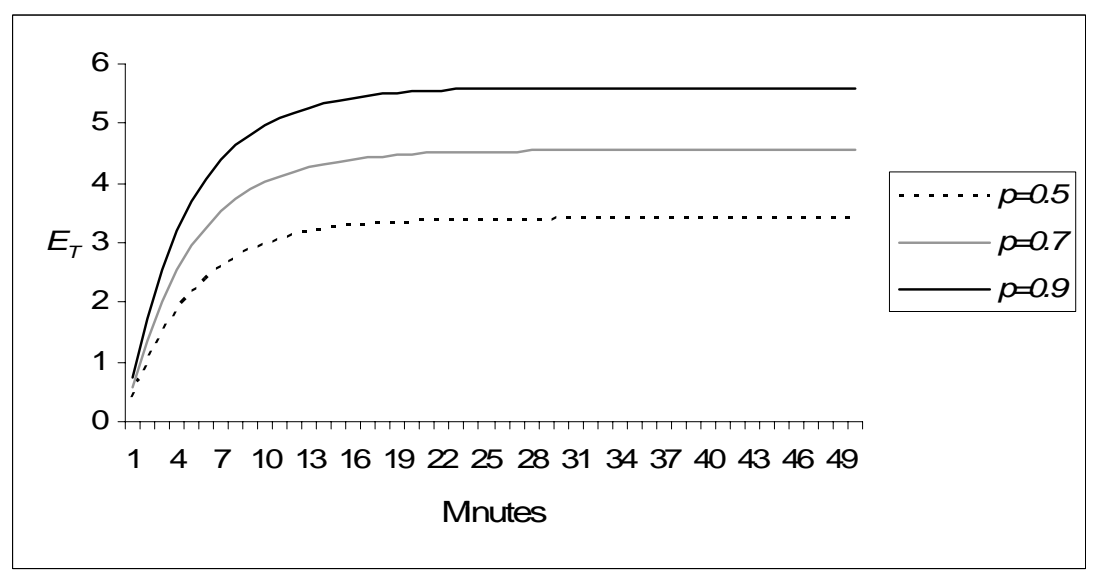

Figure 8: Expected Number of Killed Targets 
Absent situational awareness, the expected number of killed targets approaches asymptotically 3.4, 4.5 and 5.6 targets for kill probabilities .5, .7 and .9, respectively. These limit values are reached relatively fast - after about 20 minutes of operation. Figure 5.4 can help obtain some guidelines for operating the UCAVs in case they are not disposable and can be used in future operations. For example, it can identify a time $t^{*}$ at which all searching UCAVs will be programmed to abandon their mission and return to the home base.

\section{MULTIPLE UCAVS WITH IMPERFECT BDA AND LIMITED COORDINATION}

Assume now that the UCAVs have limited situational awareness, that is, $0<q_{1}, q_{2}<1$. Next we develop a continuous time Markov chain that represents our combat situation.

\section{STATES}

Let $n$ denote the number of searching UCAVs. Initially, $n=N$. A state in the model is represented by $\left(n, m_{i} ; i=0, \ldots, N-n\right)$ where $m_{i}$ indicates the number of valuable targets that are currently under attack (but have not been hit yet) by exactly $i$ UCAVs each. An absorbing state in the engagement process is of the form $\left(0, m_{0}, 0, \ldots, 0\right)$, which means that there are no UCAVs at the search stage $(n=0)$ and no UCAVs at the attack stage. The number of valuable targets killed by the UCAVs in an absorbing state is $L-m_{0}$.

Example: let $L=N=2$. There are 11 possible states: $(2,2,0,0),(1,2,0,0),(1,1,1,0)$, $(1,1,0,0),(0,2,0,0),(0,1,1,0),(0,1,0,1),(0,1,0,0),(0,0,2,0),(0,0,1,0)$ and $(0,0,0,0)$. For example, the state $(1,2,0,0)$ represents the situation where one UCAV is searching and the other UCAV is removed following a failed attack (acquired a non-valuable target or missed a valuable target or has crashed). The state $(1,1,0,0)$ represents a similar situation, however the removed UCAV successfully acquired and killed a valuable target. 


\section{STATE TRANSITIONS}

An event in this process is a detection, or a kill or a miss or a failure of the UCAV. A detection may lead to a change in the state if the target is identified as valuable, otherwise no change in state is recorded. A kill or a miss or a failure always results in a change of state. Figure 9 presents the possible transitions for the states in the above example ( $L=N=2$ ). The shaded boxes indicate absorbing (terminal) states.

In general, the following states are possible transitions from the state $\left(n, m_{i} ; i=0, \ldots, N-n\right)$.

(i) A searching UCAV has acquired a valuable target that is currently attacked by $j$ other UCAVs:

$$
\begin{aligned}
\left(n-1, m_{j}-1, m_{j+1}+1, m_{i} ; i \neq j, j+1\right) \text { with probability } \\
\frac{(1 / T) \lambda \cdot n \cdot m_{j} \cdot q_{1}}{(\lambda+\theta) \cdot n+(\mu+\theta) \cdot \sum_{i=1}^{N-n} m_{i} \cdot i} .
\end{aligned}
$$

The numerator in (8) is the rate of detection $(\lambda n) \times$ the probability of selecting a valuable target that is currently attacked by $j$ other UCAVs $\left(m_{j} / T\right) \times$ the probability of correctly identifying the valuable target $\left(q_{1}\right)$.

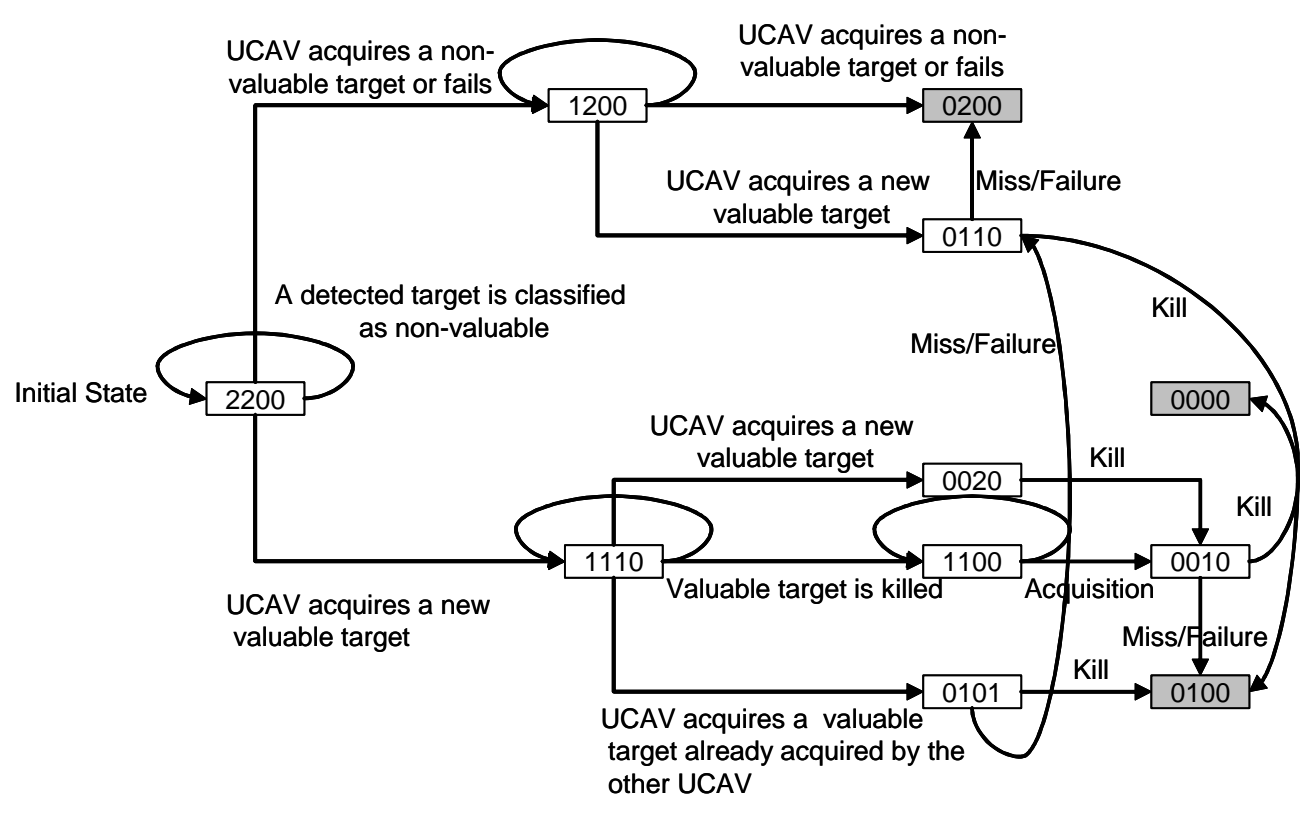


Figure 9: The State Transitions, $L=N=2$

(ii) A searching UCAV has acquired a non-valuable target or has failed (removed prematurely):

$$
\begin{aligned}
\left(n-1, m_{i} ; i=0, \ldots, N-n+1\right) \text { with probability } & \frac{(1 / T) \cdot \lambda \cdot n \cdot\left(T-\sum_{i=0}^{N-n} m_{i}\right) \cdot\left(1-q_{2}\right)+\theta n}{(\lambda+\theta) \cdot n+(\mu+\theta) \cdot \sum_{i=1}^{N-n} m_{i} \cdot i} .
\end{aligned}
$$

The numerator in (9) is the rate at which non valuable targets are acquired (= the rate of detection $(\lambda n) \times$ the probability of selecting a non-valuable target

$\left(\left(T-\sum_{i=0}^{N-n} m_{i}\right) / T\right) \times$ the probability of incorrectly identifying this target as valuable $\left.\left(1-q_{2}\right)\right)+$ the failure rate of searching UCAVs $(=\theta n)$.

(iii) A UCAV is the first to kill a valuable target that is currently attacked by $j$ UCAVs:

$$
\begin{aligned}
\left(n, m_{j}-1 ; m_{i} ; i \neq j\right) \text { with probability } \\
\qquad \frac{\mu \cdot j \cdot m_{j} \cdot p}{(\lambda+\theta) \cdot n+(\mu+\theta) \cdot \sum_{i=1}^{N-n} m_{i} \cdot i} .
\end{aligned}
$$

The numerator in (10) is the attack rate of a single $\operatorname{UCAV}(\mu) \times$ the number of UCAVs that are attacking this type of targets $\left(j \cdot m_{j}\right) \times$ the kill probability of a single $\operatorname{UCAV}(p)$.

(iv) A UCAV that is attacking a valuable target, which is currently attacked by $j$ UCAVs, is removed without completing its mission, that is, misses the target or fails during the attack: 


$$
\begin{aligned}
\left(n, m_{j-1}+1, m_{j}-1 ; m_{i} ; i \neq j-1, j\right) \text { with probability } \\
\frac{(\mu \cdot(1-p)+\theta) \cdot j \cdot m_{j}}{(\lambda+\theta) \cdot n+(\mu+\theta) \cdot \sum_{i=1}^{N-n} m_{i} \cdot i}
\end{aligned}
$$

The numerator in (11) is the rate of attacks that miss the target $\left(\mu \cdot(1-p) \cdot j \cdot m_{j}\right.$, see also (10) above) + the failure rate of attacking $\left.\operatorname{UCAVs}\left(\theta \cdot j \cdot m_{j}\right)\right)$.

(v) A detected target is classified as non-valuable and therefore passed over:

$$
\begin{aligned}
& \left(n, m_{i} ; i=0, \ldots, n\right) \text { with probability } \\
& \qquad \frac{(1 / T) \cdot \lambda \cdot n \cdot\left[\left(1-q_{1}\right) \cdot \sum_{i=0}^{N-n} m_{i}+q_{2} \cdot\left(T-\sum_{i=0}^{N-n} m_{i}\right)\right]}{(\lambda+\theta) \cdot n+(\mu+\theta) \cdot \sum_{i=1}^{N-n} m_{i} \cdot i} .
\end{aligned}
$$

The numerator in (12) is the rate at which valuable targets are misclassified as nonvaluable $(=$ detection rate $(\lambda n) \times$ probability of selecting a valuable target $\left(\sum_{i=0}^{N-n} m_{i} / T\right) \times$ the probability for type- 1 error $\left.\left(1-q_{1}\right)\right)+$ the rate at which nonvaluable targets are classified correctly as such $\left(\lambda \cdot n \cdot q_{2} \cdot\left(T-\sum_{i=0}^{N-n} m_{i}\right) / T\right)$.

Suppose now that the UCAVs can share information and coordinate their attacks. Specifically, we assume that during the attack stage a UCAV sends out a signal that marks ("highlights") its target. The signal, which is set off when the UCAV is removed, may be received by any searching UCAV with a fixed probability $r$. The signals from the various UCAVs are independent. Thus, a searching UCAV that detects a target that is currently attacked by $j$ other UCAVs avoids it without further investigation with probability $1-(1-r)^{j}$. Notice that if $r=1$ then no incidents of multiple acquisitions (attacks) can occur. The transition rates shown above change only for cases (i) and (v): 
(i) A searching UCAV has acquired a live (valuable) target that is already attacked by j other UCAVs:

$$
\begin{aligned}
\left(n-1, m_{j}-1, m_{j+1}+1, m_{i} ; i \neq\right. & j, j+1) \text { with probability } \\
& \frac{(1 / T) \lambda \cdot n \cdot m_{j} \cdot q_{1} \cdot(1-r)^{j}}{(\lambda+\theta) \cdot n+(\mu+\theta) \cdot \sum_{i=1}^{N-n} m_{i} \cdot i} .
\end{aligned}
$$

(v) A target is passed over (is valuable but recognized as being acquired by other UCAVs or is classified as non-valuable or is non-valuable):

$$
\begin{aligned}
& \left(n, m_{i} ; i=0, \ldots, N-n\right) \text { with probability } \\
& \qquad \frac{(1 / T) \cdot \lambda \cdot n \cdot\left[\sum_{i=0}^{N-n} m_{i}\left((1-r)^{i} \cdot\left(1-q_{1}\right)+1-(1-r)^{i}\right)+q_{2}\left(T-\sum_{i=0}^{N-n} m_{i}\right)\right]}{(\lambda+\theta) \cdot n+(\mu+\theta) \cdot \sum_{i=1}^{N-n} m_{i} \cdot i} .
\end{aligned}
$$

All other transitions ((ii) - (iv)) remain the same.

To keep the model tractable, we assume that this transfer of attack information does not apply to non-valuable targets. Otherwise we need to keep track also of the number of non-valuable targets that are being attacked by $i$ UCAVs, which leads to a considerable expansion of the state dimension. If the number of non-valuable targets is relatively high compared to the numbers of valuable targets and UCAVs, and if the specificity of the sensor $q_{2}$ is reasonably high, then we can assume that instances of multiple acquisitions of non-valuable targets are highly unlikely. In particular, we assume that there are practically no incidents where a UCAV avoids acquiring a certain non-valuable target solely because it receives a signal from another UCAV that has already acquired (erroneously) that non-valuable target. Another assumption that leads to the same transition probabilities is that $r \approx 0$ for acquisitions of nonvaluable targets (e.g., a UCAV realizes rather quickly that it has acquired a nonvaluable target and sets the signal off immediately).

\section{MEASURES OF EFFECTIVENESS}

To evaluate the relative effects of design and operational parameters we define four measures of effectiveness (MOE): 
- Expected relative effectiveness $\left(E_{M}\right)$ is the ratio between the expected number of killed valuable targets and their initial number. This MOE represent the effectiveness of the attack. Formally,

$$
E_{L}=\frac{E[X]}{L}
$$

where $X$ is the number of killed valuable targets.

- Expected relative efficiency $\left(E_{N}\right)$ is the ratio between the expected number of killed valuable targets and the initial number of UCAVs in the attack pack. This MOE represent how efficient is the mission. Formally,

$$
E_{N}=\frac{E[X]}{N} .
$$

- Probability of attaining the mission objective $\left(P_{\alpha}\right)$ is the probability that at least a fraction $\alpha$ of the $L$ valuable targets are killed. This MOE represents tactical or operational objectives, as set by the mission commander. Clearly, this MOE is non-trivial only if $N \geq \alpha L$. Formally,

$$
P_{\alpha}=\operatorname{Pr}(X \geq \alpha L) \text {. }
$$

In addition to the three MOEs we compute also the expected duration of a mission $E_{\text {Time }}$. The results are obtained by utilizing computational procedures of absorbing Markov chains (e.g., Minh (2000)).

\section{ANALYSIS}

The time parameters in our base case are as in Section 5: $\lambda=0.2, \mu=2$ and $\theta=0.01$.

The sensitivity, specificity and kill probabilities are $q_{1}=0.7, q_{2}=0.8$ and $p=0.8$, respectively. These values represent only a reasonable reference point for the technical and operational parameters of UCAVs since most of these vehicles are still in the development phase. Even if some relevant data do exist, it would be most likely classified. Notwithstanding this limitation, the ensuing sensitivity analysis provides insights into tradeoffs among the parameters of the vehicle and the combat scenario. The base case scenario comprises a pack of $N=8 \mathrm{UCAVs}$ that engages a total of $T=$ 12 targets, out of which $L=8$ are valuable. We first assume no coordination, that is $r$ $=0$. The expected number of killed valuable targets is 4.32 with engagement effectiveness and efficiency of $E_{L}=E_{N}=0.54$. The probability of attaining the mission objective - at least $40 \%$ of the valuable targets killed - is $P_{0.4}=0.77$. The expected 
duration of the operation is $E_{\text {Time }}=30 \mathrm{~min}$. If the UCAVs are fully coordinated then $E_{L}=E_{N}=0.55, P_{0.4}=0.78$ and $E_{\text {Time }}=30.4 \mathrm{~min}$. Clearly, in the base case, coordination has no significant effect; the changes in the MOEs values are negligible.

Next we investigate the impact of various parameters on the values of the MOEs.

\section{(a) Detection and Attack Rates}

For a fixed failure rate of $\theta=0.01$ (base case) Figures $10-13$ present the effect of the detection rate $(\lambda)$ and the attack rate $(\mu)$ on the expected relative effectiveness $E_{N}$ and on the probability of attaining a mission objective of $40 \%$ killed valuable targets $P_{0.4}$. Since $L=N$, the expected relative effectiveness is also the expected relative efficiency. Figures 10 and 11 apply to the case where there is no coordination among the UCAVs $(r=0)$, while Figures 12 and 13 apply to the case of full coordination ( $r=$ $1)$.

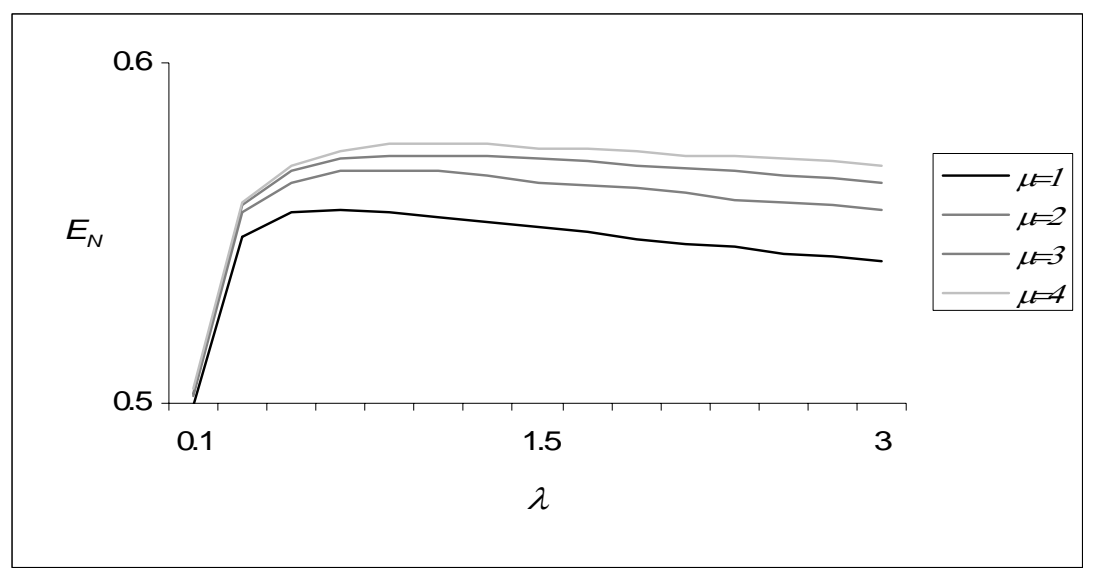

Figure 10: The Effect of Detection Rate on the Expected Relative Effectiveness (Efficiency), $r=0$. 


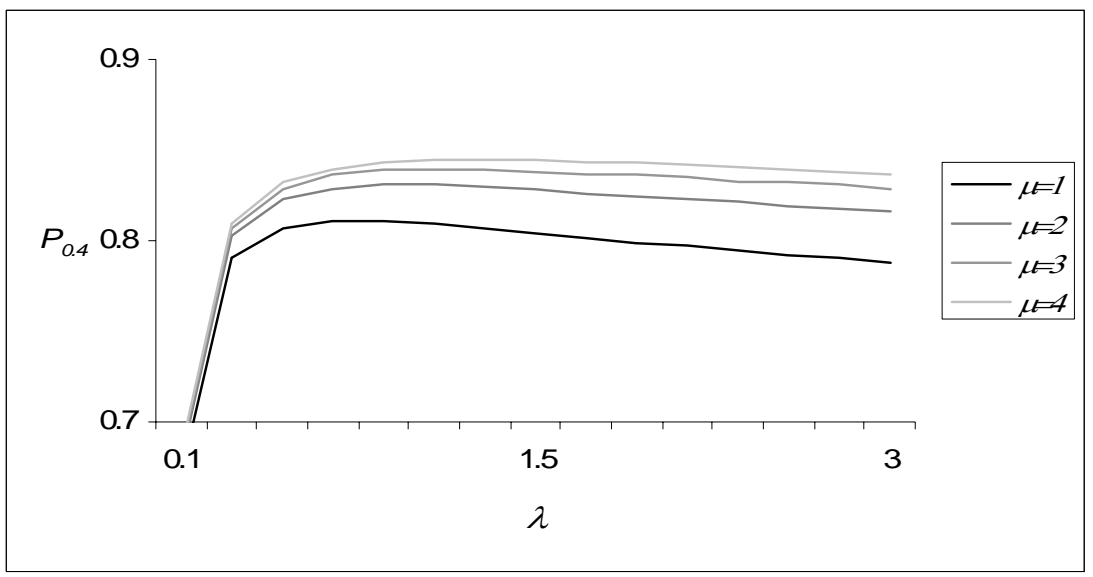

Figure 11: The Effect of Detection Rate on the Probability of Attaining 40\% Killed Valuable Targets, $r=0$.

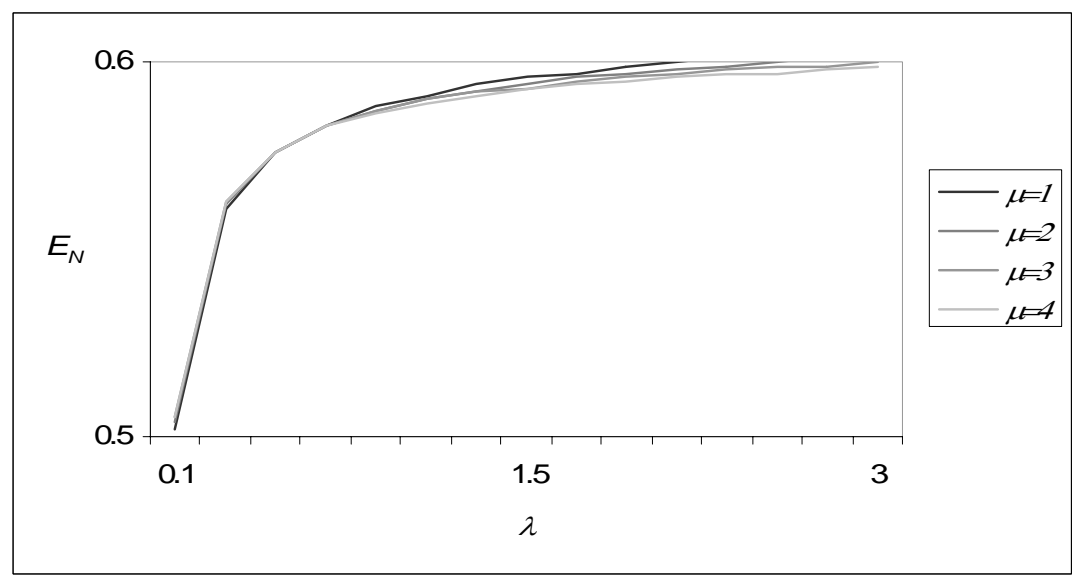

Figure 12: The Effect of Detection Rate on the Expected Relative Effectiveness (Efficiency), $r=1$.

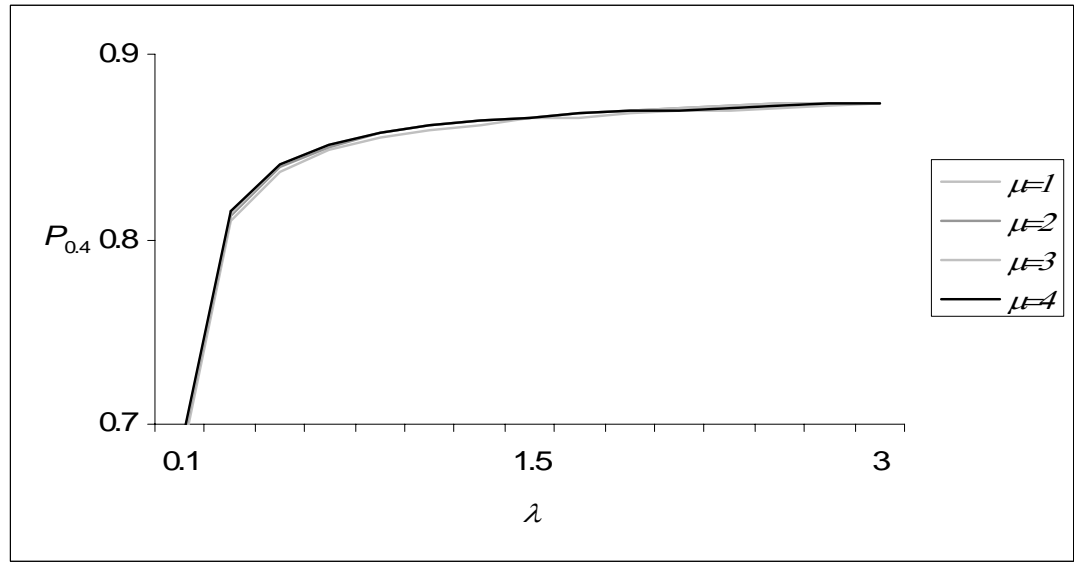

Figure 13: The Effect of Detection Rate on the Probability of Attaining 40\% Killed Valuable Targets, $r=1$. 
In all four charts the mean detection time of a UCAV ranges between 10 minutes ( $\lambda=0.1$ ) and 20 seconds $(\lambda=3)$. Both MOEs $-E_{N}$ and $P_{0.4}$ - are computed for four

mean attack times that range from 1 minute $(\mu=1)$ to 25 seconds $(\mu=4)$. In the case of no coordination $(r=0)$, shorter attack times result in better performance of the UCAVs with respect to both MOEs. This conclusion is quite intuitive for cases of relatively high sensitivity, specificity and kill probability. Shorter attack times reduce the possibility of redundant multiple attacks. The observation that higher detection rate may be counter-effective, as displayed by the unimodal plots in Figures 10 and 11 , is less intuitive. The monotonic increasing part for small values of $\lambda$ represents a race between the detection and failure processes; increasing detection rate decreases loitering time and therefore also the chances for failure. The monotonic decreasing part for larger values of $\lambda$ is explained by exactly the same arguments used above for explaining the positive effect of increasing $\mu$; shorter detection time relative to the attack time implies more opportunities for simultaneous acquisitions that lead to multiple attacks. The effect of $\theta$ is discussed later on. In the case of perfect coordination ( $r=1)$ there can be no multiple acquisitions and therefore higher detection rate is always better. Since the attack time is very short compared to the $\operatorname{MTBF}\left(\theta^{-1}\right)$ of the UCAVs, perfect coordination implies that the effect of the attack rate $\mu$ is negligible.

Note that the graphs of $E_{N}$ and $P_{0.4}$ have similar shapes. For brevity we display from now on mostly results regarding $E_{N}$ or $E_{L}$.

\section{(b) Sensitivity, Specificity and Kill Probability}

An interesting question regarding the UCAV's sensor capabilities is: which property is more important, sensitivity or specificity? Recall that higher sensitivity means lower probability for type I error (misclassifying a valuable target), while higher specificity implies lower probability for type II error (misclassifying a non valuable target). Figures 14 and 15 show the effect of changing the sensitivity and specificity of the sensor, respectively. The results are displayed for 4 values of kill probability: 0.6, 0.7, 0.8 and 0.9. All other parameters are set at their base case values. 


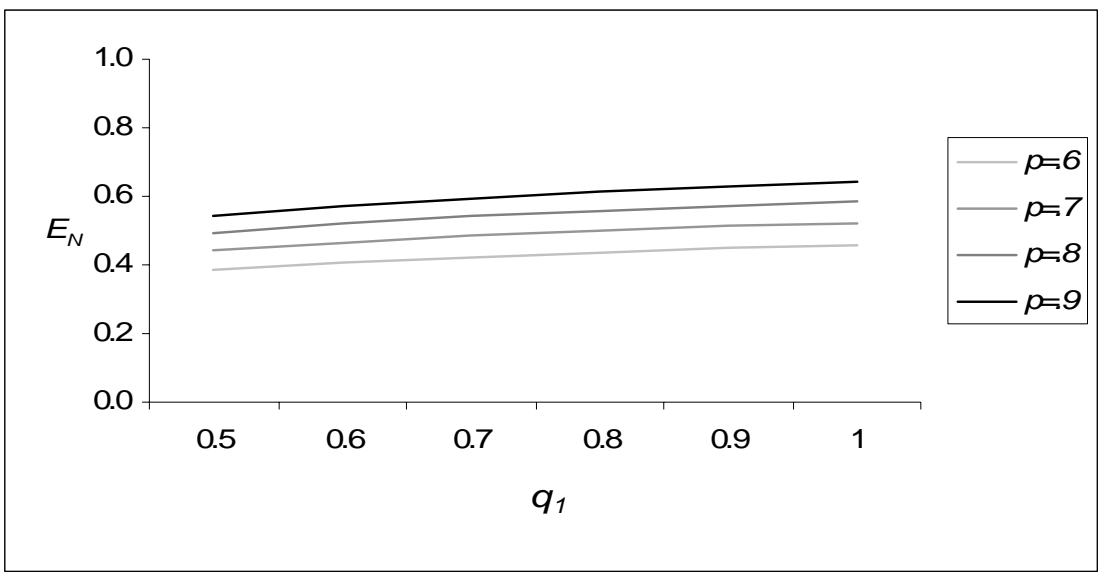

Figure 14: The Effect of Sensor Sensitivity on the Expected Relative Effectiveness.

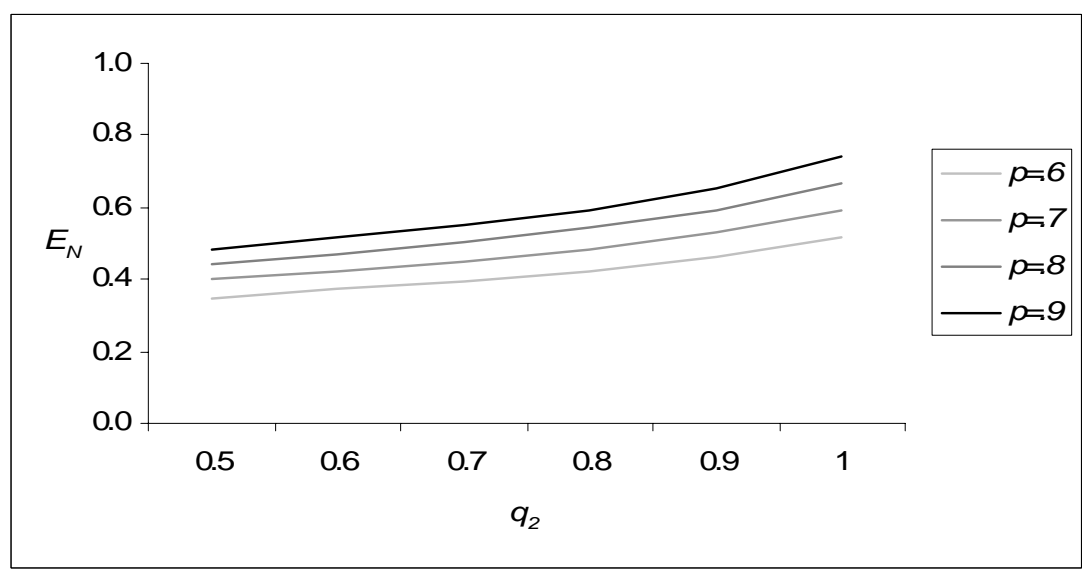

Figure 15: The Effect of Sensor Specificity on the Expected Relative Effectiveness.

Note that while $E_{N}$ is a concave function of the sensor's sensitivity, it is a convex function of its specificity. When we move $q_{1}$ from 0.5 to $1, E_{N}$ increases by $18 \%$ for all values of $p$. The corresponding increase in $E_{N}$ when $q_{2}$ varies is $47 \%$ for $p=0.6$ and $53 \%$ for $p=0.9$. We conclude that the effect of specificity on the outcome of the attack is stronger than sensitivity, and this effect becomes more significant for higher values of kill probability. Specifically, suppose that the decision is either to increase the sensitivity of the sensor by $20 \%$ from its current base case value, or to increase by a similar rate its specificity. Recall that for the base case $E_{N}=0.54$. If $q_{1}$ is increased by $20 \%$ then $E_{N}=0.56$, while if $q_{2}$ is increased by $20 \%$ then $E_{N}=0.64$. The choice is clear; in order to increase the effectiveness of the attack one should invest in improving the specificity of the UCAV's sensor, rather than its sensitivity. This conclusion applies to our case of disposable (or one-weapon) UCAVs where a false 
positive error is irreversible. This may not be the case if the UCAV has multiple weapons and the mission is not time-critical. The recommendation to invest in better specificity is enhanced by other measures of merit such as the human and political cost of attacking a wrong target (e.g., the bombing of the Chinese embassy in Belgrade by NATO forces in 1999).

\section{(c) Failure Rate and Coordination}

Arguably, UCAVs' coordination can be effective only if the attack stage is long compared to the search stage. If it is short, then multiple acquisitions are very unlikely and therefore there is no practical need for coordination. It is shown next that the failure rate may affect the benefit the pack gains from coordination. Let $L=6$, and suppose that the attack time is four time shorter than the detection time, which is set at its base case value. This situation may represent a standoff attack. The failure rate ranges between 0 (no failure during the mission) to $0.1(\mathrm{MTBF}=10 \mathrm{~min}$ ). Figure 16 presents the expected relative effectiveness for $r=0$ and $r=1$. All other parameters are set at their base case values.

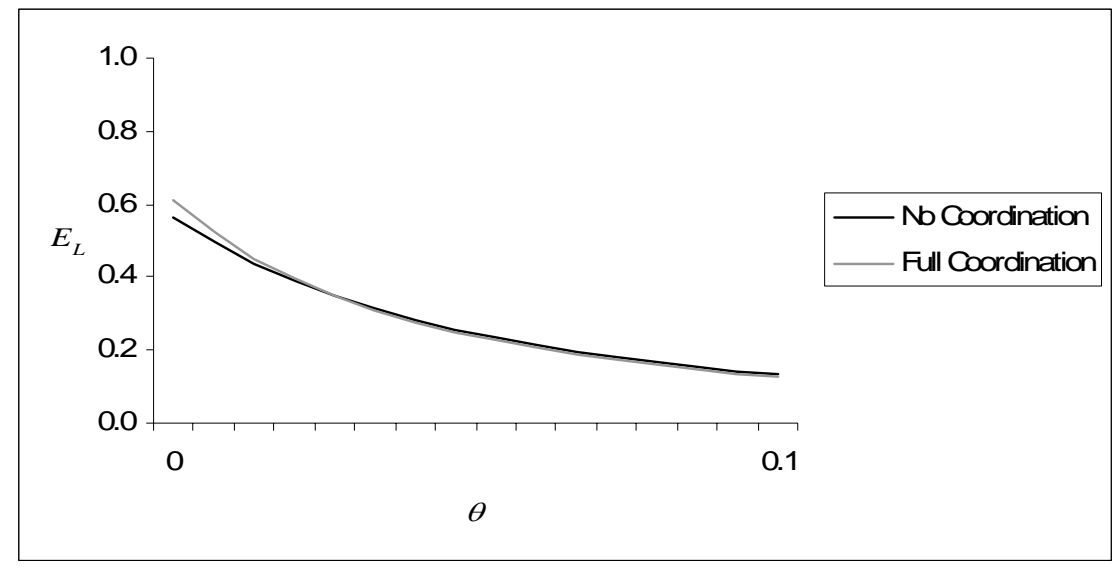

Figure 16: The Effect of Failure Rate and Coordination on the Expected Relative Effectiveness.

As one would expect, the effectiveness of the UCAVs decreases as the failure rate increases. Note that even in this extreme scenario, where conditions are relatively favorable for effective coordination, the effect is minute. Moreover, while for smaller failure rates full coordination is somewhat more effective than no coordination, the opposite is true for larger failure rates for which coordination actually reduces the mission effectiveness. The latter counter-intuitive observation is due to the fact that if 
UCAVs pass over targets, they prolong their stay in the target area and therefore increase their chances to be intercepted before staging their attack.

Another way to avoid multiple acquisitions is to employ the UCAVs sequentially rather than simultaneously as a pack. This tactical solution to multiple acquisition problem leads to a different Markov model that is based on the probabilities given in (2) above. Taking once again $L=6, \mu=\lambda / 4$ and the rest of the parameters at their base case values, Figure 17 presents the value of the expected relative effectiveness $E_{L}$ for three cases: No coordination, perfect coordination and sequential engagement. These values are computed as functions of the specificity probability $q_{2}$.

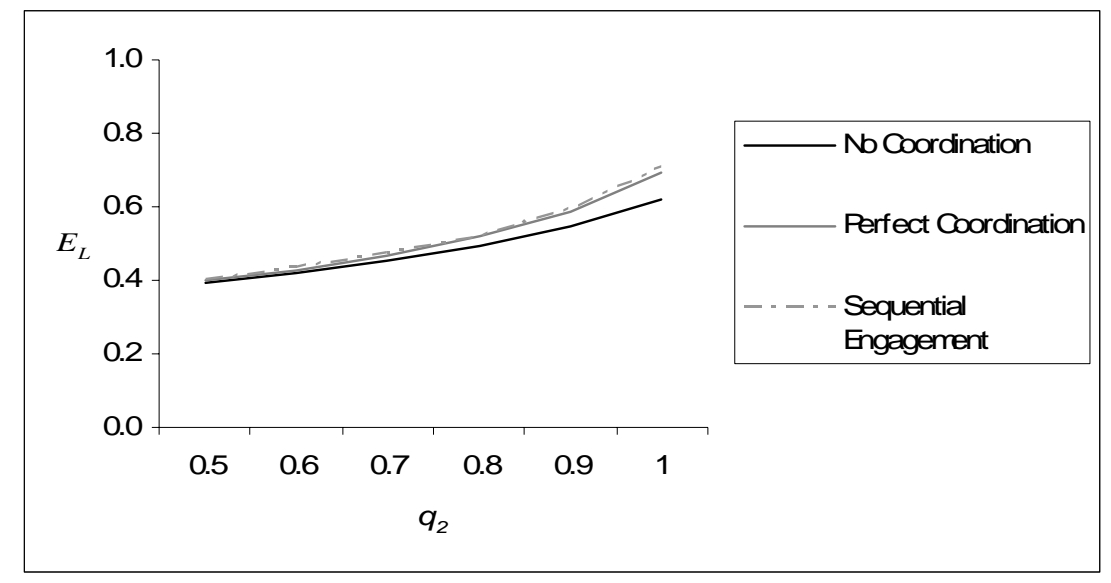

Figure 17: The Effect of Eliminating Multiple Acquisitions

For poor to moderate specificity the three graphs coincide. For high specificity, eliminating multiple acquisitions, either by a design features (coordination) or tactics (sequential engagement) has some effect. The effect is similar in both cases, with a slight advantage to the tactical solution, which is applicable only for non time-critical targets.

\section{(d) Scenario Parameters}

So far we have analyzed the effect of parameters that are associated with the design of the UCAVs. Figures 18 and 19 display the effect of the scenario. Figure 18 presents the value of $E_{L}$ when the number of valuable targets $L$ and the specificity probability $q_{2}$ vary in the target area. Note that besides being a design parameter, specificity is 
also a scenario parameter that may depend on the clutter in the target area. Figure 19 displays the combined effect of $L$ and the number of UCAVs $N$. The MOE here is $P_{0.4}$, which represents a specific tactical objective.

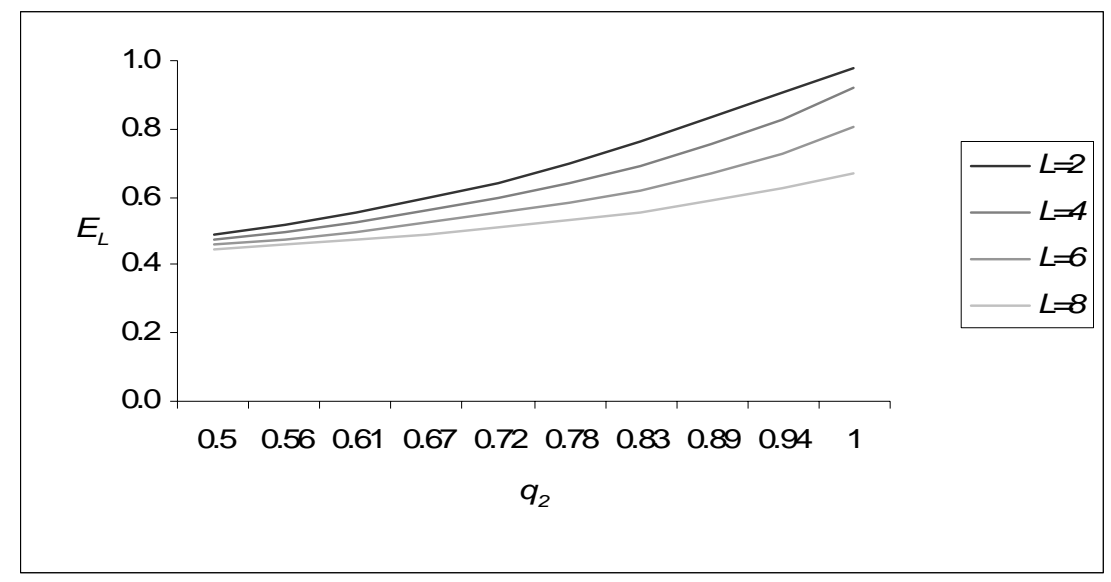

Figure 18: The Effect of the Number of Valuable Targets

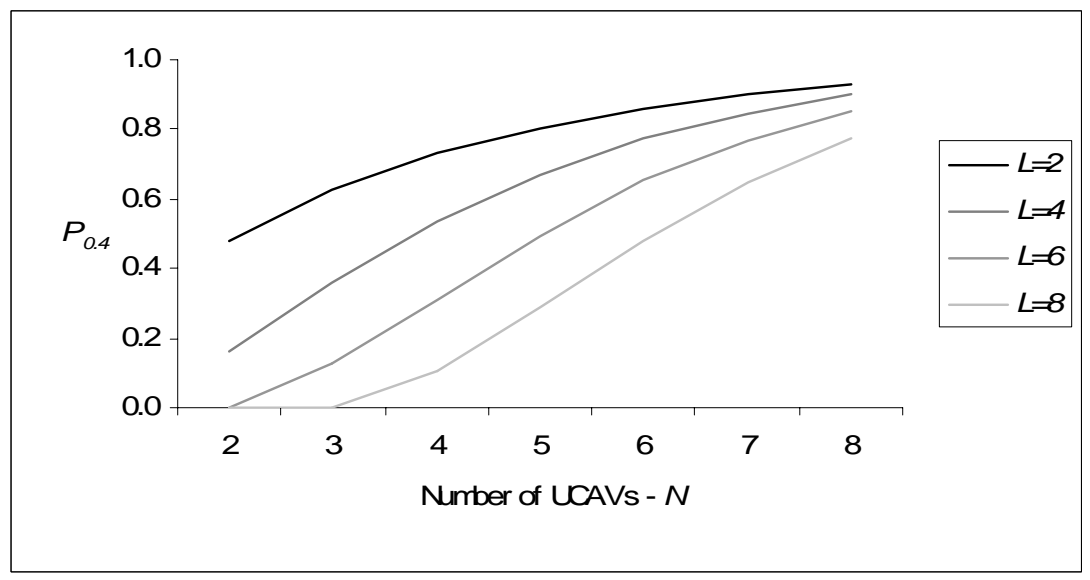

Figure 19: Probability of Attaining 40\% Killed Valuable Targets as a Function of $M$ and $N$

From Figure 18 we see once again the effect of specificity. At low specificity, the rate of killed valuable targets is relatively insensitive to their number. At high specificity this rate decreases with the number of targets, as one would expect.

Figure 19 examines the impact of the number of valuable targets on the engagement performance from another angle. For small number of UCAVs the $40 \%$ attrition probability is very sensitive to the number of targets. This sensitivity diminishes as $N$ gets larger. Note that Figure 19 may be used also as a decision support tool for 
mission planning. For example, if there are four valuable targets in the target area, then in order to attain the mission objective - two killed targets - with probability of at least 0.8 , then the pack must contain at least seven UCAVs. This can be seen by observing the point at which the graph corresponding to $L=4$ crosses the 0.8 threshold.

\section{SUMMARY, CONCLUSIONS AND FUTURE RESEARCH}

In this paper we explore several design and operational aspects of employing a pack of autonomous UCAVs against valuable targets that are imbedded among other, nonvaluable targets. Utilizing newly developed analytic probability models, we evaluate the effect of key design and operational parameters on the performance of the pack. First, it is shown that under reasonable assumptions memory is a redundant property. The processing capacity in the UCAV brain should be utilized to other tasks such as enhanced recognition capability. Second, based on a transient model, inter-temporal behavior of the system is explored and some insights regarding mission duration and maximum allowable loitering time are obtained. It is shown that detection rate is a major factor in determining the duration of the operation. Finally, in Section 6 we implement a large-scale continuous-time Markov model to analyze the effect of weapon coordination on multiple acquisitions, and the effect of BDA on multiple kills. The two main conclusions from the analysis are: (1) attack coordination among UCAVs is largely an insignificant feature for the scenarios analyzed, and (2) specificity of the UCAV's sensor is more important than its sensitivity. The first conclusion is true as long as the valuable targets are homogeneous. It essentially says that the random uniform and independent selection is the right thing to do when engaging uniform targets. If among the valuable targets there are some that are more noticeable or attractive then targeting coordination may improve the engagement performance. The case of non-homogeneous targets is left for future research. The second conclusion tells us that avoiding non-valuable targets is more beneficial than picking correctly valuable ones. This observation, which at first glance may look a little odd, is quite logical. Type I error by a UCAV (passing over a valuable target) can be rectified later on. Type II error (acquiring and attacking non-valuable target) cannot. 
The models described in this paper are limited to homogeneous targets, homogeneous UCAVs and to the engagement rules specified. Another limitation is the assumption that all the temporal random variables are exponential. While this assumption is reasonable for the failure and detection processes, the attack time is probably not well represented by a constant failure-rate (CFR) distribution. Accordingly, the models

presented in this paper may be extended to account for non-homogeneous targets, multiple types of UCAVs and more general time CDFs (e.g., non-exponential attack times). Another interesting and potentially important extension is to incorporate in the models decision rules where the UCAVs manifest some level of cognitive capability. Specifically, in reality both sensitivity and specificity probabilities may depend on the time a UCAV spends investigating a target. This aspect is not captured in our models and may lead to interesting optimization models.

\section{REFERENCES}

Airforce Technology, 2005, "Predator RQ-1 / MQ-1 / MQ-9 Unmanned Aerial Vehicle (UAV), USA”, http://www.airforce-technology.com/projects/predator/.

Aviv, Y., and Kress, M., 1997, "Evaluating the Effectiveness of Shoot-Look-Shoot Tactics in the Presence of Incomplete Damage Information”, Military Operations Research, V. 3, No. 1, pp 79-89.

Chandler, P. R., Pachter, M., Nygard, K. E., and Swaroop, D., 2002, “Cooperative Control for Target Classification”, in R. Murphy and P. Pardalos, Ed. Cooperative Control and Optimization, Kluwer Academic Publishers, 2002, pp 1-19.

Gillen, D., and Jacques, D. R., 2002, “Cooperative Behavior Schemes for Improving the Effectiveness of Autonomous Wide Area Search Munitions”, Kluwer Academic Publishers, 2002, pp 95-120.

Glazebrook, K., and Washburn, A. R., 2004, “Shoot-Look-Shoot: a Review and Extension”, Operations Research, V. 52, pp 454-463. 
Jacques, D. R., 2002, “Modeling Considerations for Wide Area Search Munition Effectiveness Analysis”, Proceedings of the 2002 Winter Simulation Conference, pp 878-886.

Jane’s, 2000a, “Briefing - Autonomous Weapons”, Jane’s Defense Weekly, V. 33/6, February 9, 2000.

Jane’s, 2000b, “Germany Funds Final Phase of TAIFUN UAV”, Jane’s Defense Weekly, V. 33/17, April 26, 2000.

Jane’s, 2002, “Air-to-Surface Missiles”, Jane’s Air-Launched Weapons, V. 40, 23 April, 2002.

Kress, M., 1991, “A Two-on-One Stochastic Duel with Maneuvering and Fire Allocation Tactics”, Naval Research Logistics, V. 38, No. 3, pp 303-313.

Kress, M., and Talmor, I., 1999, “A New Look at the 3:1 Rule of Combat Through Markov Stochastic Lanchester Models”, Journal of the Operational Research Society, V. 50, No. 7, pp 733-744.

Lockheed Martin, 2004, “Autonomous Wide Area Search Munition”, Defense Update - International Online Defense Magazine, Issue 5, 2004, http://www.defenseupdate.com/products/a/awasm.htm.

Manor, G., and Kress, M., 1997, “Optimality of the Greedy Shooting Strategy in the Presence of Incomplete Damage Information”, Naval Research Logistics, V. 44, No. 7, pp 613-622.

Minh, D. L., 2000, Applied Probability Models, Duxbury Press, pp 98-107.

Richards, A., Bellingham, J., Tillerson, M, and How, J., 2002, “Co-ordination and Control of Multiple UAVs”, Proceedings of the AIAA Guidance, Navigation and Control Conference, Monterey CA. 
Yost, K., and Washburn, A. R., 2000, “Optimizing assignment of Air-to-Ground Assets and BDA Sensors”, Military Operations Research, V. 5, No. 2, pp 7791.

\section{APPENDIX}

Acquisition probabilities in the full-memory (M) and no-memory (NM) cases.

\begin{tabular}{|c|c|c|c|c|c|c|c|c|c|}
\hline \multirow{3}{*}{$q_{1}$} & \multirow{3}{*}{$q_{2}$} & \multicolumn{4}{|c|}{$\frac{\lambda}{\theta}=20$} & \multicolumn{4}{|c|}{$\frac{\lambda}{\theta}=3$} \\
\hline & & \multicolumn{2}{|c|}{$\mu=\lambda$} & \multicolumn{2}{|c|}{$\mu=10 \lambda$} & \multicolumn{2}{|c|}{$\mu=\lambda$} & \multicolumn{2}{|c|}{$\mu=10 \lambda$} \\
\hline & & $N M$ & $M$ & $N M$ & $M$ & $N M$ & $M$ & $N M$ & $M$ \\
\hline \multirow{3}{*}{0.5} & 0.5 & .22 & .22 & .23 & .23 & .11 & .11 & .15 & .14 \\
\hline & 0.7 & .30 & .30 & .31 & .31 & .14 & .14 & .18 & .17 \\
\hline & 0.9 & .48 & .47 & .50 & .49 & .18 & .17 & .23 & .22 \\
\hline \multirow{3}{*}{0.7} & 0.5 & .28 & .28 & .29 & .29 & .15 & .15 & .19 & .19 \\
\hline & 0.7 & .37 & .38 & .39 & .40 & .18 & .18 & .23 & .23 \\
\hline & 0.9 & .56 & .56 & .58 & .59 & .23 & .22 & .29 & .29 \\
\hline \multirow{3}{*}{0.9} & 0.5 & .33 & .34 & .34 & .35 & .18 & .18 & .23 & .24 \\
\hline & 0.7 & .43 & .44 & .45 & .46 & .22 & .22 & .28 & .28 \\
\hline & 0.9 & .61 & .63 & .64 & .66 & .27 & .27 & .34 & .35 \\
\hline
\end{tabular}

Table A1: Acquisition Probability - Proportion of Valuable Targets $=1 / 4$ 


\begin{tabular}{|c|c|c|c|c|c|c|c|c|c|}
\hline \multirow{3}{*}{$q_{1}$} & \multirow{3}{*}{$q_{2}$} & \multicolumn{4}{|c|}{$\frac{\lambda}{\theta}=20$} & \multicolumn{4}{|c|}{$\frac{\lambda}{\theta}=3$} \\
\hline & & \multicolumn{2}{|c|}{$\mu=\lambda$} & \multicolumn{2}{|c|}{$\mu=10 \lambda$} & \multicolumn{2}{|c|}{$\mu=\lambda$} & \multicolumn{2}{|c|}{$\mu=10 \lambda$} \\
\hline & & $N M$ & $M$ & $N M$ & $M$ & $N M$ & $M$ & $N M$ & $M$ \\
\hline \multirow{3}{*}{0.5} & 0.5 & .43 & .43 & .45 & .45 & .23 & .22 & .29 & .29 \\
\hline & 0.7 & .53 & .53 & .55 & .55 & .26 & .25 & .33 & .32 \\
\hline & 0.9 & .68 & .67 & .71 & .71 & .30 & .29 & .38 & .37 \\
\hline \multirow{3}{*}{0.7} & 0.5 & .51 & .52 & .54 & .54 & .28 & .28 & .36 & .36 \\
\hline & 0.7 & .61 & .61 & .63 & .64 & .31 & .31 & .46 & .46 \\
\hline & 0.9 & .74 & .74 & .77 & .78 & .36 & .36 & .49 & .49 \\
\hline \multirow{3}{*}{0.9} & 0.5 & .57 & .58 & .60 & .60 & .33 & .33 & .45 & .45 \\
\hline & 0.7 & .68 & .69 & .69 & .70 & .36 & .36 & .49 & .50 \\
\hline & 0.9 & .79 & .80 & .81 & .82 & .41 & .41 & .55 & .55 \\
\hline
\end{tabular}

Tale A2: Acquisition Probability - Proportion of Valuable Targets = 1/2 


\begin{tabular}{|c|c|c|c|c|c|c|c|c|c|}
\hline \multirow{3}{*}{$q_{1}$} & \multirow{3}{*}{$q_{2}$} & \multicolumn{4}{|c|}{$\frac{\lambda}{\theta}=20$} & \multicolumn{4}{|c|}{$\frac{\lambda}{\theta}=3$} \\
\hline & & \multicolumn{2}{|c|}{$\mu=\lambda$} & \multicolumn{2}{|c|}{$\mu=10 \lambda$} & \multicolumn{2}{|c|}{$\mu=\lambda$} & \multicolumn{2}{|c|}{$\mu=10 \lambda$} \\
\hline & & $N M$ & $M$ & $N M$ & $M$ & $N M$ & $M$ & $N M$ & $M$ \\
\hline \multirow{3}{*}{0.5} & 0.5 & .65 & .65 & .68 & .67 & .34 & .33 & .44 & .43 \\
\hline & 0.7 & .71 & .71 & .75 & .74 & .36 & .35 & .46 & .46 \\
\hline & 0.9 & .79 & .79 & .83 & .82 & .38 & .38 & .50 & .49 \\
\hline \multirow{3}{*}{0.7} & 0.5 & .71 & .72 & .75 & .75 & .40 & .40 & .52 & .51 \\
\hline & 0.7 & .77 & .77 & .80 & .81 & .42 & .42 & .54 & .54 \\
\hline & 0.9 & .83 & .83 & .87 & .87 & .45 & .44 & .58 & .57 \\
\hline \multirow{3}{*}{0.9} & 0.5 & .76 & .76 & .79 & .80 & .45 & .45 & .58 & .58 \\
\hline & 0.7 & .80 & .81 & .84 & .84 & .47 & .47 & .60 & .60 \\
\hline & 0.9 & .86 & .86 & .90 & .90 & .49 & .49 & .63 & .63 \\
\hline
\end{tabular}

Tale A3: Acquisition Probability - Proportion of Valuable Targets = 3/4 